\title{
Promoter Hypermethylation-mediated Inactivation of LRRC4 in
} Gliomas

\author{
Zuping Zhang ${ }^{1,2}$, Dan $\mathrm{Li}^{1}$, Minghua Wu ${ }^{* 1}$, Bo Xiang ${ }^{1}$, Li Wang ${ }^{1}$, Ming Zhou ${ }^{1}$, \\ Pan Chen ${ }^{1}$, Xiaoling $\mathrm{Li}^{1}$, Shourong Shen ${ }^{3}$ and Guiyuan $\mathrm{Li}^{* 1}$
}

Address: ${ }^{1}$ Cancer Research Institute, Central South University, Changsha 410078, Hunan, PR China, ${ }^{2}$ Department of Parasitology, Central South University, Changsha 410078, Hunan, PR China and ${ }^{3}$ The Third Affiliated Hospital of Xiang Ya School of Medicine, Central South University, Changsha 410078, Hunan, PR China

Email: Zuping Zhang - zhangzp74@126.com; Dan Li - dennis_k@126.com; Minghua Wu* - wuminghua554@yahoo.com.cn; Bo Xiang - bobxiang4@hotmail.com; Li Wang - wangli120@21cn.com; Ming Zhou - zhouming2001@163.com; Pan Chen - chenpan520123@163.com; Xiaoling Li - lixl@xysm.net; Shourong Shen - Ssr-35403@163.com; Guiyuan Li* - ligy@xysm.net

* Corresponding authors

Published: 3 November 2008

BMC Molecular Biology 2008, 9:99 doi:10.1 186/1471-2199-9-99

This article is available from: http://www.biomedcentral.com/I47/-2/99/9/99

(C) 2008 Zhang et al; licensee BioMed Central Ltd.

This is an Open Access article distributed under the terms of the Creative Commons Attribution License (http://creativecommons.org/licenses/by/2.0), which permits unrestricted use, distribution, and reproduction in any medium, provided the original work is properly cited.
Received: 18 June 2008

Accepted: 3 November 2008

\begin{abstract}
Background: Leucine-rich repeat C4 protein (LRRC4) is a new member of the leucine-rich repeat (LRR) superfamily. It is not only a brain-specific gene but also a novel candidate for tumor suppression. LRRC4 inactivation is commonly found in glioma cell lines and primary glioma biopsies. However, little is known about the mechanism controlling LRRC4 expression. In a previous study, we did not find any genetic alteration in LRRC4 in primary glioma, which led us to explore an alternative mechanism underlying this phenomenon.
\end{abstract}

Methods: In the present paper, we cloned the LRRC4 promoter with characteristics of a CPG island by luciferase reporter assay. Then, the CPG methylation status around the LRRC4 promoter region in glioma cell lines and primary gliomas was examined by methylation-specific PCR and bisulfite DNA sequencing. In order to demonstrate a functional association between LRRC4 promoter methylation and its gene inactivation, we performed DNA demethylation analysis with two human glioma cell lines using methylation-specific PCR and RT-PCR.

Results: The sequence spanning positions -835 to -293 relative to the translation start site was identified as the LRRC4 promoter; this sequence is a TATA- and CAAT- less, high GC content region. It was found that $L R R C 4$ promoter activity is strongly suppressed after treatment with Sssl methylase in vitro. Furthermore, LRRC4 promoter methylation was observed by methylationspecific PCR in two glioma cell lines and all 30 primary glioma specimens, but not in normal brain tissue. Bisulfite DNA sequencing showed that most of the $C_{p} G$ sites were located around the $L R R C 4$ promoter methylated in glioma cells and tissues, but not in normal brain tissue. In addition, the methylase inhibitor 5-Aza-2'-deoxycytidine could induce LRRC4 mRNA expression and LRRC4 promoter partial demethylation in SFI26 and SF767 glioma cells.

Conclusion: Methylation-mediated inactivation of LRRC4 is a frequent and glioma-specific event, and it may be a potential biomarker for diagnosis or prognosis, or serve as a therapeutic target. 


\section{Background}

Gliomas are the most common malignant tumors in the adult central nervous system and account for 50 to $60 \%$ of primary brain tumors. These cancers exhibit a relentless malignant progression characterized by widespread invasion throughout the brain, and thus usually result in a poor prognosis [1]. Although multiple genetic alterations are involved in the development and progression of malignant gliomas [2,3], epigenetic silencing of wild-type tumor suppressor genes via aberrant promoter hypermethylation has also been shown to occur [4-6]. Aberrant promoter methylation of $\mathrm{CpG}$ island-associated genes is a common epigenetic alteration associated with the inactivation of tumor suppressor and other genes in human cancers [7-9]. Unmethylated in normal tissues, promoters of these genes can become methylated de novo in cancer cells. This change is accompanied by alterations in histone modification and chromatin conformation, rendering the promoter transcriptionally inert [10].

Such epigenetic mechanisms have been implicated in the inactivation of several key regulators of the cell cycle $(R B$, p16INK4A, p73), DNA repair $\left(O^{6} M G M T\right)$, apoptosis (DAP kinase), angiogenesis (THBS1), and invasion (TIMP3) in glioma [11]. Recently, novel hypermethylated genes in glioma have been identified using a candidate gene approach or by a genome-wide screening method. The former revealed that genes such as EMP3, TMS1/ASC, SLC5A8, hMLH and PTEN are frequently targeted for DNA methylation-mediated silencing in glioma $[4,5,12,13]$. A genome-wide screen using a combined approach of pharmacologic inhibition of epigenetic modifications and gene expression microarrays also revealed that several novel genes are subject to aberrant hypermethylation in glioma [14,15]. Thus, aberrant methylation events have become critical to our understanding of the initiation and progression of human brain malignancies and may serve as a biomarker for diagnosis, prognosis and susceptibility to treatment.

Leucine-rich repeat $\mathrm{C} 4$ protein (LRRC4) is a new member of the leucine-rich repeat (LRR) superfamily located at 7q31-32 [16]. It was found to be predominantly expressed in normal brain tissue and involved in early nervous system development and differentiation [17], but the expression of LRRC4 was absent in several malignant glioma cell lines [18]. Similarly, it was absent or significantly downregulated in $87.5 \%$ of primary glioma biopsies [16]. More importantly, LRRC4 had the potential to suppress tumorigenesis of U251 malignant glioma cells in vivo and cell proliferation in vitro [19]. Recent studies show that LRRC4 can block U251 cells in $\mathrm{G}_{0} / \mathrm{G}_{1}$ and induce U251 cell-growth arrest and differentiation by down-regulating the ERK/Akt/NF-кB, STAT3 and JNK2/p-c-Jun/p53 signaling pathways [18]. Therefore, the loss of LRRC4 function may be an important event in the progression of gliomas and may act as a novel candidate for tumor suppression. However, little is known about the mechanism of LRRC4 expression loss or down- regulation in glioma cell lines and biopsies. No known studies have found genetic alterations in the LRRC4 coding sequence in glioma biopsies or cell lines [18]. This lack of findings led us to explore an alternative mechanism underlying inactivation of LRRC4 in glioma.

In the present study, we cloned the LRRC4 promoter with characteristics of a CpG island. Then, CpG methylation status around the LRRC4 promoter region in glioma cell lines and primary gliomas was examined by methylationspecific PCR and bisulfite DNA sequencing. In order to demonstrate a functional association between LRRC4 promoter methylation and its gene inactivation, we performed DNA demethylation analysis with two human glioma cell lines using methylation-specific PCR and RTPCR.

\section{Methods \\ Cell lines and tumor samples}

Thirty fresh tumor samples were collected after informed consent was obtained from patients who underwent brain operations for glioma at Xiangya Hospital (Hunan, People's Republic of China). The samples were snap-frozen immediately following resection and stored in liquid nitrogen until processing. The 17 male and 13 female patients were aged from 17 to 68 years (mean age at registration $=42$ years). Tumors were graded and classified according to the World Health Organization (2007), including astrocytoma (grade I (1), grade II-III (17)), oligodendroglioma (grade II (5)), oligoastrocytoma (grade II (3)), and glioblastoma (grade IV (4)) [33]. For comparison, normal human tissues from patients without cancer were obtained at the time of autopsy.

Human glioblastoma-derived cell lines SF126 and SF767 were obtained from the Cell Research Institute of Peking Union Medical College (Peking, China) and cultured in minimal essential medium (MEM). Cos7 and Hela were obtained from American Type Culture Collection and maintained in Dulbecco's modified Eagle's medium (DMEM). All cells were supplemented with 10\% heatinactivated fetal bovine serum (FBS), $100 \mathrm{U} / \mathrm{mL}$ penicillin and $100 \mathrm{ug} / \mathrm{mL}$ streptomycin and cultured at 37C with $5 \% \mathrm{CO}_{2}$.

\section{Cloning and analysis of the LRRC4 5 ' upstream regulatory region}

The LRRC4 promoter region in the 5' end of humans was predicted using the PromoterInspector and PromoterScan programs. The CpG island was found using CpGplot from the European Molecular Biology Open Software Suite. To 
obtain the 5' flanking region of the LRRC4 gene, PCR amplification was performed on human genomic DNA (forward primer 5'-ATTGGTACCGGCGAGCTCACAGGGCAGGG-3', reverse primer 5'-CTTAGATCTCTGGAGAAGGAGGTGGGGAG-3'). After an initial denaturation step $\left(94^{\circ} \mathrm{C}\right.$ for $5 \mathrm{~min}$ ), the PCR reactions were carried out for 32 cycles at $94^{\circ} \mathrm{C}$ for $30 \mathrm{sec}, 62^{\circ} \mathrm{C}$ for $30 \mathrm{sec}$, and $72^{\circ} \mathrm{C}$ for $2 \mathrm{~min}$, with a final extension of $10 \mathrm{~min}$ at $72^{\circ} \mathrm{C}$. The PCR product was purified using a gel Purification Kit and cloned into the T/A cloning vector pGEMT-Easy (Promega). Positive clones of pT/A-2475/-101 were isolated and sequenced.

\section{Luciferase-reporter plasmid constructs and assay}

The 5' upsteam regulatory region of the LRRC4 gene was subcloned into the KpnI and Bgl II restriction sites of the pGL3-enhancer vector (Promega). Construct naming is based on the position of the promoter fragments (pGL32475/-101). Four deletion constructs of the LRRC4 promoter region were created (pGL3-1483/-101, pGL3-835/101, pGL3-293/-101 and pGL3-835/-293), originating from the construct pGL3 -2475/-101 by PCR amplification using the primers listed in Table 1. Sense primers for generating the reporter constructs described above contained an adaptor with a KpnI restriction site (GGTACC) at the $5^{\prime}$ end. Anti-sense primers contained an adaptor with a Bgl II restriction site (AGATCT). The promoter fragments were then subcloned into the KpnI/Bgl II sites of the pGL3 enhancer vector and sequenced.

Transfection was performed with Lipofectamine $e^{\mathrm{TM}}$ Reagent (Invitrogen, Carlsbad, CA). $5 \times 10^{5}$ cells were seeded in each well of 24-well tissue plates. When cells reached 50$80 \%$ confluence, they were cotransfected using $1 \mu \mathrm{g}$ of each DNA construct in pGL3-enhancer and $0.25 \mu \mathrm{g}$ SV40 $\beta$-galactosidase vector for normalizing transfection efficiency per well according to manufacturer's instructions. Firefly luciferase activity was measured in cell lysates $48 \mathrm{~h}$ after transfection by using the Luciferase Assay System (Promega) and a luminometer. $\beta$-galactosidase activity was measured in cell lysates by the $\beta$-galactosidase Enzyme Assay System (Promega). Experiments were repeated at least three times with three replicates per sample for each experiment. Results are normalized against $\beta$ galactosidase activity.

\section{Methylation of report plasmid constructs in vitro}

pGL3-835/-293 report plasmid constructs were methylated in vitro by SssI methylase (New England Biolabs) treatment following the manufacturer's instructions. Cells were transfected by methylated or mock-methylated constructs as described above.

\section{DNA extraction and Bisulfite modification}

Genomic DNA from cells and tissues was prepared using a DNA Extraction Kit (TaKaRa) according to manufacturer's instructions. Five hundred nanograms of genomic DNA was modified and purified using an EZ DNA Methylation-Gold Kit (ZYMO RESEARCH), following the manufacturer's protocol. Modified DNA was used immediately or stored at $-80^{\circ} \mathrm{C}$ for up to six months.

\section{Methylation-Specific PCR}

The methylation-specific PCR primers were designed according to the promoter-active DNA sequence using Methyl Primer Express v1.0. Modified DNA was amplified by two different primer pairs specific to the unmethylated (U) and methylated (M) LRRC4 promoter sequences, respectively. For the methylated $(M)$ sequence, the forward and backward primers were 5'-AGCGTAGTATTTAGCGAGTGC-3' and 5'-TAAACCCTAACACCGACTCG$3 '$.

For the unmethylated (U) sequence, the primers were forward 5'-GGGAGTGTAGTATTTAGTGAGTGT-3' and backward 5'-TAAACCCTAACACCAACTCACTC-3'. PCR amplification was performed for a total of 35 cycles with an annealing temperature of $58^{\circ} \mathrm{C}$. Methylation specific PCR products were analyzed by a $2 \%$ agarose gel and stained with ethidium bromide.

\section{Bisulfite Sequencing}

In order to cover the whole promoter region of LRRC4, two PCR regions were amplified using primers that

Table I: Primer Pairs Used for Generating LRRC4 Promoter Constructs pGL3-2475/-101, pGL3-I483/-101, pGL3-835/-101, pGL3-293/$10 I$ and $p G L 3-835 /-293$

\begin{tabular}{ll}
\hline pGL3-2475/-101 & Forward: 5'-ATTGGTACCGGCGAGCTCACAGGGCAGGG-3' \\
& Reverse: 5'-CTTAGATCTCTGGAGAAGGAGGTGGGGAG-3' \\
pGL3-1483/-101 & Forward: 5'-ATCGGTACCAGTATGCGTCAGCAGTACATTCACG-3' \\
& Reverse: 5'-CTTAGATCTCTGGAGAAGGAGGTGGGGAG-3' \\
PGL3-835/-101 & Forward:5'-ATCGGTACCAACACCTCCTCTTAACTCTCGCC-3' \\
& Reverse: 5'-CTTAGATCTCTGGAGAAGGAGGTGGGGAG-3' \\
PGL3-293/-101 & Forward:5'-ATTGGTACCTGCTTTCCTGCCTTCCTTCC-3' \\
& Reverse: 5'-CTTAGATCTCTGGAGAAGGAGGTGGGGAG-3' \\
PGL3-835/-293 & Forward:5'-ATCGGTACCAACACCTCCTCTTAACTCTCGCC-3' \\
& Reverse: 5'-ATTAGATCTGCACTGGCGTGGTGTCCTTA-3'
\end{tabular}


avoided CpG sites. For Region 1, primers were 5'-GYGGATTGGAGAATTGATTT TT-3' and 5'-AACTATACAAATACATACCCCCCCC-3'. For Region 2, primers were 5'GAGGGGGGGGTATGTATTTGTATAGT-3' and 5'CCCACCCTCAAAACAAACCC TC-3'. The PCR amplification was performed for a total of 38 cycles with annealing temperature of $56^{\circ} \mathrm{C}$. PCR products were gel-purified and cloned into the T/A cloning vector pGEMT-Easy (Promega). Ten subclones were isolated and identified by double digestion and sequencing.

\section{5'-Aza-dC treatment of cell lines}

The human glioblastoma-derived cell lines SF126 and SF767 were grown for 4 days in the presence of various concentrations of 5-Aza-dC (2.5, 5, 10 and $15 \mu \mathrm{M})$. Fresh drug was added every $24 \mathrm{~h}$. RNA and DNA were separately isolated.

\section{RT-PCR}

RNA was isolated from harvested cells with Trizol (Invitrogen) reagent and then treated with DNase (Roche) to eliminate contaminated DNA. Reverse transcription of the RNA was performed according to the instructions of Promega. To amplify LRRC4, $2 \mu \mathrm{l}$ of cDNA was used for each PCR using the primers, 5'-CAACTTGGCCCACAAT AACC-3' (forward) and 5'-CATCCGACCCTCAGAAATGT-
3' (reverse). The primers for GAPDH were 5'-GTCAGTGGTGGACCTGACC T-3' (forward) and 5'-AGGGGAGATTCAGTGTGGTG-3'(reverse). The GAPDH primers were added to the PCR at the end of the tenth cycle as control experiments. Ten microliters of each reaction was then run on $2 \%$ agarose gel and stained with ethidium bromide.

\section{Results}

Cloning and analysis of the $\mathbf{5}$ ' upstream regulatory region To clone the LRRC4 promoter region, a database was searched against the human genomic DNA using LRRC4 (Genbank accession No. AF196976) as the query http:// www.ncbi.nlm.nih.gov/BLAST to reveal the 5'upstream sequence of the LRRC4 gene. Several bioinformatics tools were used to identify the potential promoter region of the LRRC4 gene. A 605-bp region spanning positions -814 to -210 was identified as the potential promoter region of the LRRC4 gene by using a PromoterInspector [20], whereas a 251-bp region located from -788 to -538 was identified as the LRRC4 promoter by using a PromoterScan program [21]. We detected two CpG islands that spanned positions -2151 to -433 and -366 to -101 using the EMBOSS CpGplot program [22] (Figure 1). Finally, a genomic DNA fragment that spanned positions -2475 to 101 relative to the initiation codon ATG of the LRRC4

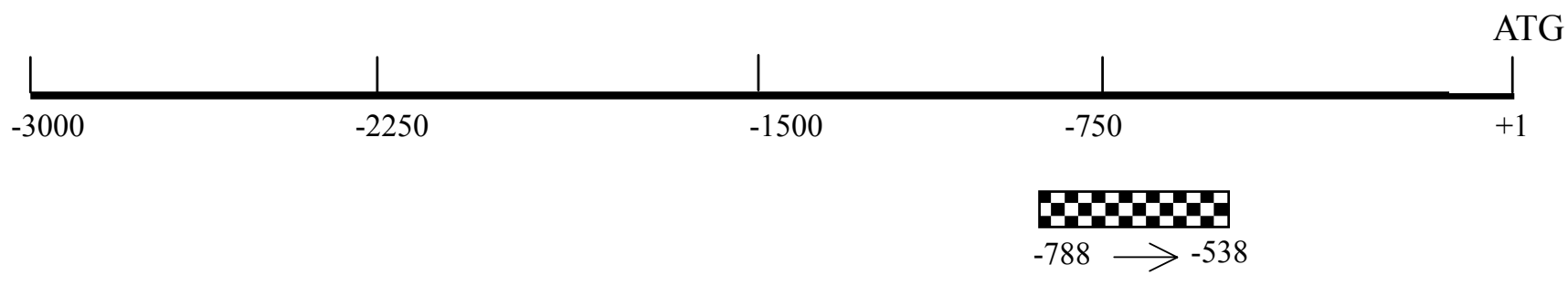

$251 \mathrm{bp}$ promoter region

PromoterScan program
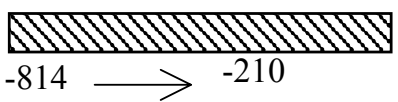

605 bp promoter region

PromoterInspector Program
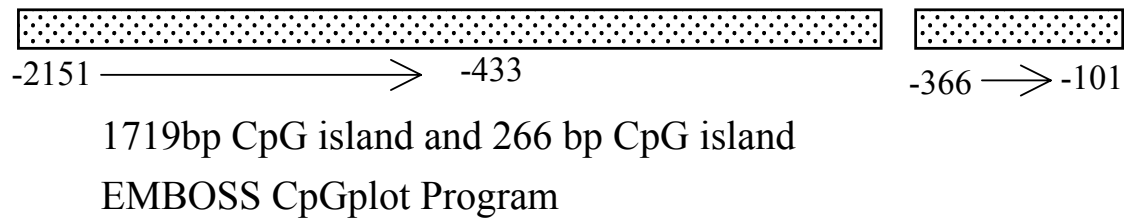

$1719 \mathrm{bp} \mathrm{CpG}$ island and $266 \mathrm{bp} \mathrm{CpG}$ island

EMBOSS CpGplot Program

Figure I

Analysis of the LRRC4 5' upstream regulatory region by bioinformatics. The regions showing the putative promoter activity and CpG islands are shown as a square box, a hatched box and dotted boxes, respectively. The translation start site is position $+I$ and the rest of the sequence is numbered relative to it. 
gene was amplified by PCR. The PCR product was cloned into the T/A cloning vector pGEMT-Easy (Promega) (Data not shown).

\section{Functional analysis of the LRRC4 promoter by 5 ' upstream deletion}

To identify the promoter region of the LRRC4 gene, five constructs of progressive deletion spanning positions 2475 to -101 were generated (pGL3-2475/-101, pGL3 1483/-101, pGL3-835/-101 and pGL3-293/-101) and cloned to upstream of the luciferase reporter gene in the pGL3-enhancer vector (Promega). Transient transfection experiments were carried out using Cos7 and Hela cells, respectively. The luciferase activity driven by LRRC4 promoter constructs was measured $48 \mathrm{~h}$ after transfection. Expression levels were corrected for variable transfection efficiencies by cotransfection with a plasmid directing the $\beta$-galactosidase expression.

As shown in Figure 2, in Cos7 and Hela cells, luciferase expression driven by the constructs pGL3-2475/-101, pGL3-1483/-101, pGL3-835/-101 and pGL3-835/-293 exhibited similarly high levels, but the reporter driven by construct pGL3-293/-101 showed little luciferase activity. Thus, the sequence spanning position -835 to -293 relative to the translation start site of LRRC4 functions as a promoter. MethPrimer program analysis shows that the LRRC4 promoter region has high G/C content (approximately 70\%) and characteristics of a CpG island [23]. In

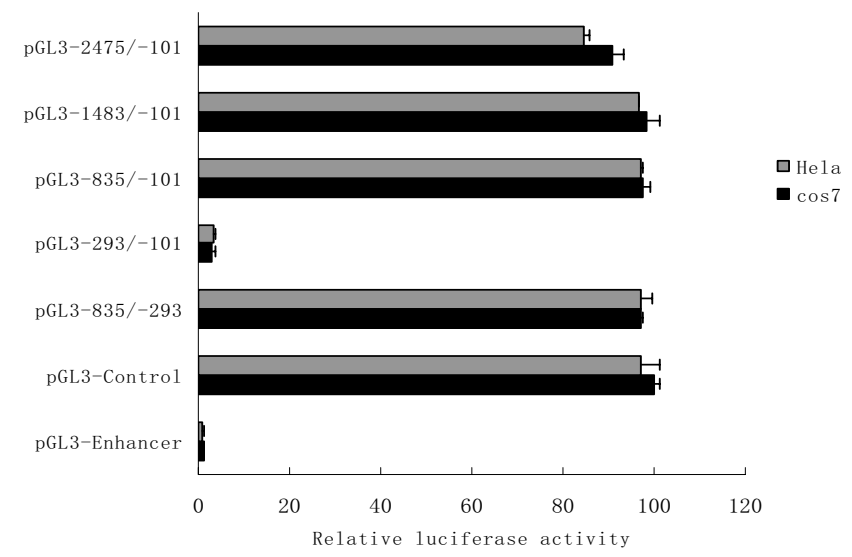

\section{Figure 2}

Deletion analysis of the LRRC4 promoter. Luciferase activity of the deleted constructs in Cos7 and Hela cells. Luciferase activity in Cos7 and Hela cells is represented by the black histogram and gray histogram, respectively. All transfection experiments were repeated at least three times, and luciferase activity was normalized by using $\beta$-galactosidase as an internal standard. Mean data \pm standard errors are shown. In both cell lines, the construct from -835 to -293 was sufficient to mediate maximal promoter activity. addition, this promoter region has no TATA box or CAAT box.

\section{DNA methylation suppresses LRRC4 promoter activity}

The presence of a CPG island suggested the possibility that the gene might be regulated through changes in the methylation status, which have been shown to cause gene silencing [24,25]. To analyze the effects of DNA methylation on promoter activity, LRRC4 promoter reporter construct pGL3-835/-293 was treated with or without SssI methylase to methylate the promoter in vitro. In vitromethylated or mock-methylated LRRC4 promoter constructs were transfected into Cos7 and Hela cells along with SV40 $\beta$-galactosidase vector. Firefly luciferase activity was measured in cell lysates as shown in Figure 3A. It can be seen that LRRC4 promoter activity was strongly suppressed by methylation in both cell lines. The same result was verified in SF126 and SF767 cell lines (Figure 3B).

\section{Methylation of the LRRC4 promoter in glioma cell lines and biopsies}

Our previous study showed that the expression of LRRC4 was absent in glioma cell lines SF126 and SF767 [18]. Moreover, DNA sequence analysis failed to identify any mutation in the LRRC4 coding region, except for one Single Nucleotide Polymorphisms site hat does not affect amino acid sequences [18]. To clarify the mechanism of LRRC4 inactivation in these two cell lines, methylationspecific PCR was used to examine the methylation status of the LRRC4 promoter. Both SF767 and SF126 cell lines showed methylation of the LRRC4 promoter. The methylated sequence was obtained and is shown in Figure 4A.

To investigate whether aberrant methylation of the LRRC4 in glioma cell lines reflects an epigenetic event occurring in primary glioma, we next examined 30 primary glioma biopsies and three tissue specimens of normal brain for LRRC4 methylation. Some methylation analyses are shown in Figure 4B. It was demonstrated that the LRRC4 promoter was free from methylation in the three normal brain tissue samples, but was methylated to different extents in the 30 primary gliomas.

\section{Bisulfite sequence analysis of promoter region of LRRC4} To determine a more detailed map of the methylation in the LRRC4 promoter, we performed bisulfite sequencing around the promoter region of the LRRC4 gene in some of the glioma biopsies and cell lines studied above. In order to cover the whole promoter region of LRRC4, two PCR regions were amplified using primers that avoid $\mathrm{CpG}$ sites. Region 1 spans -934 to -529 relative to the LRRC4 translation start site, including $48 \mathrm{CpG}$ sites. Region 2 covers -550 to -197 relative to the LRRC4 translation start site, including $21 \mathrm{CpG}$ sites. Bisulfite sequencing of 10 individual clones of PCR products of both Region 1 and Region 


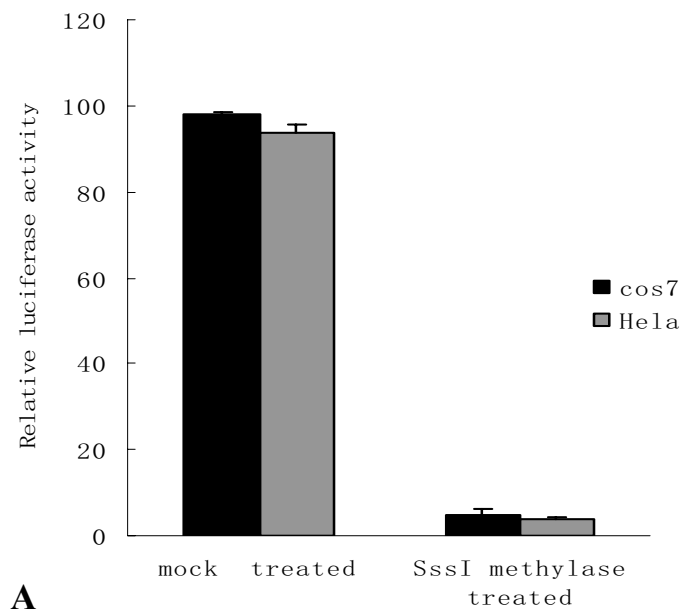

A

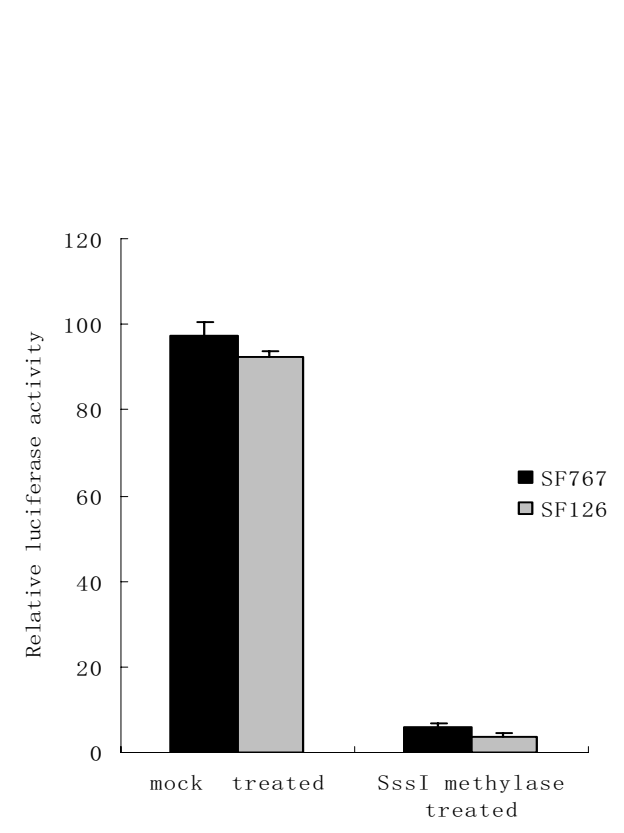

B

Figure 3

LRRC4 promoter activity is suppressed by methylation in vitro. A. Luciferase activity assays showing LRRC4 promoter repression in response to methylation. Methylated (Sssl) or mock-methylated LRRC4 promoter luciferase reporter constructs were transfected into Cos7 and Hela cells. Luciferase activity was measured in cell extracts $48 \mathrm{~h}$ after transfection. Luciferase activity in Cos7 and Hela cells is represented by the black and gray histograms, respectively. Luciferase activity was normalized using $\beta$-galactosidase as an internal standard. The results are shown as the mean \pm SD of 3 independent experiments. B.LRRC4 promoter activity suppressed by methylation was verified in glioma cell lines SFI 26 and SF767.

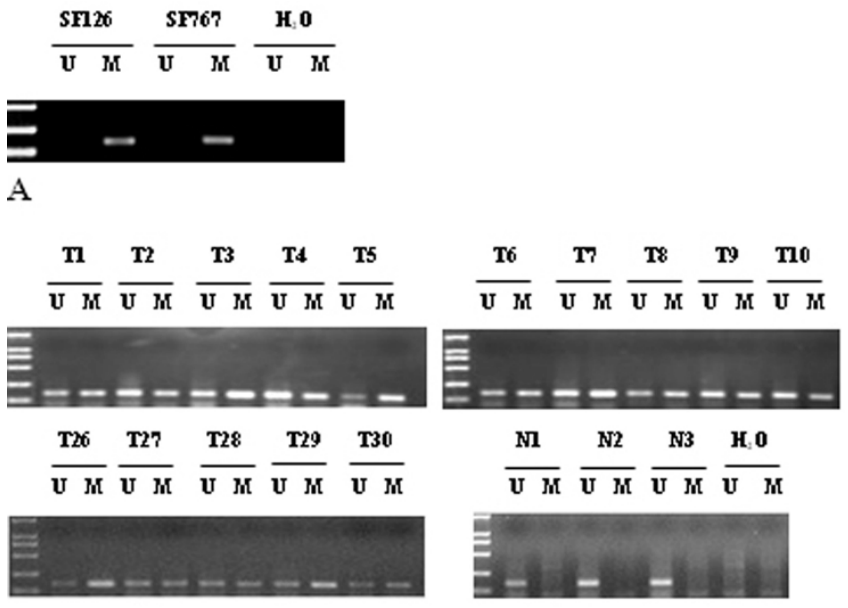

B

\section{Figure 4}

Methylation of the LRRC4 promoter in glioma. A. Methylation of the LRRC4 promoter in glioma cell lines SFI 26 and SF767. DNA from the indicated glioma cell lines was bisulfite-modified and analyzed for methylation of the LRRC4 promoter by methylation-specific PCR analysis. The presence of a PCR product in lane $U$ indicates unmethylated $L R R C 4$.

The presence of a PCR product in lane $M$ indicates the methylated $L R R C 4$. B. Schematic presentation of methylation analysis of the $L R R C 4$ gene promoter using MSP in tumor samples (T) taken from patients with glioma and three normal brain tissue samples $(\mathrm{N})$.

2 from primary glioma biopsies (T5 and T10) and cell lines (SF126 and SF767) revealed densely methylated CpGs within the promoter regions compared to normal brain tissue (Figure 5). Whereas not all CpGs around the LRRC4 promoter are methylated, most of them are in SF126 and SF767 glioma cell lines. However, in the primary glioma tissue samples (T5 and T10), the methylation pattern appeared much more heterogeneous and varied in density in different clones of the same sample. This may be due to mixed cellularity in tissue samples.

\section{5-Aza-dC induced the expression of LRRC4 in glioma cell lines}

In order to demonstrate a functional association between LRRC4 promoter methylation and its gene inactivation, a DNA demethylating agent, 5-Aza-2'-deoxycytidine (5Aza-dC), was used to treat SF126 and SF767 cell lines. By RT-PCR analysis, LRRC4 expression was detected after treatment with 5-Aza-dC in both cell lines (Figure 6A). Moreover, LRRC4 expression increased with increasing dosage of 5-Aza-dC. LRRC4 expression was highest when induced by $15 \mu \mathrm{M}$ 5-Aza-dC in SF767 cells, but in SF126 cells LRRC4 expression was highest when induced by 10 $\mu \mathrm{M}$ 5-Aza-dC. 
Region 1

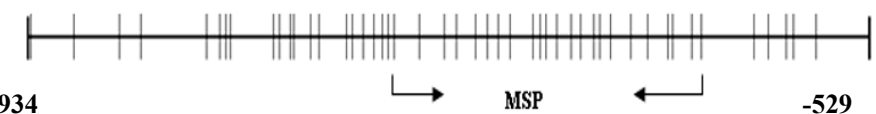

$-934$

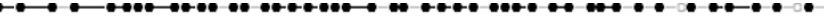

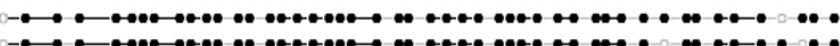

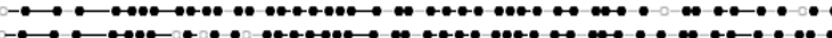

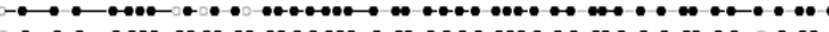

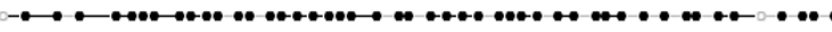

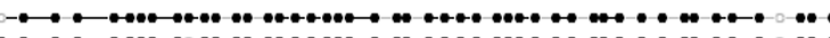

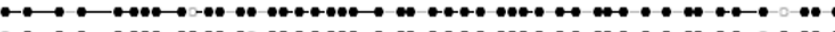

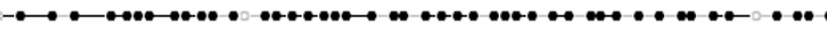

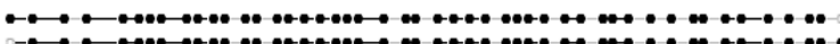

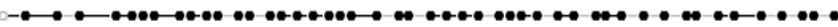

SF126

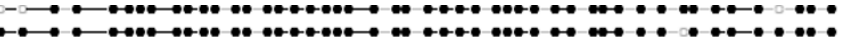

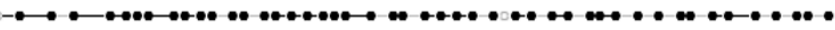

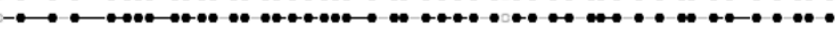
$-0_{-}-0_{0}$

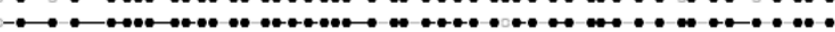

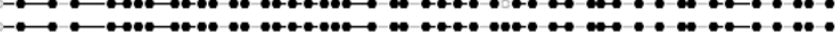
-

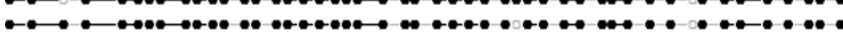
$\rightarrow$

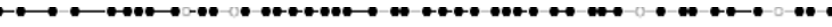

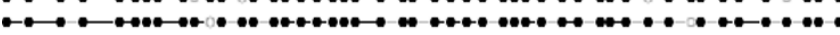

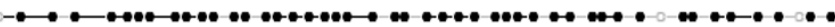

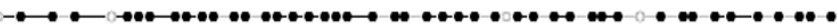

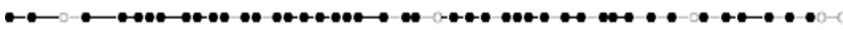

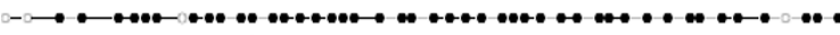

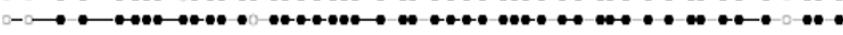

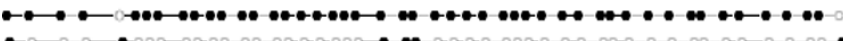

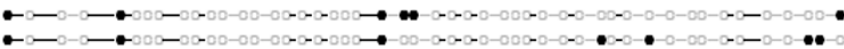

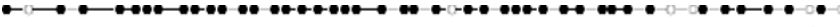

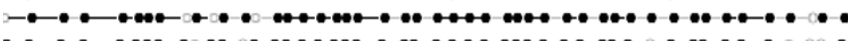

T10

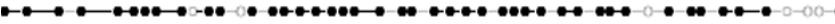

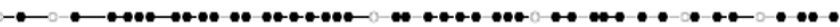

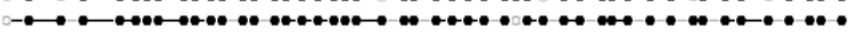

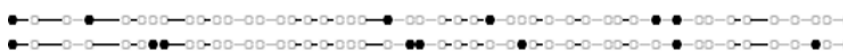

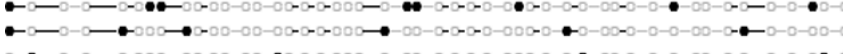

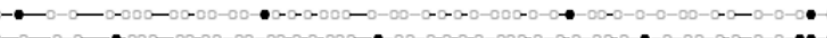

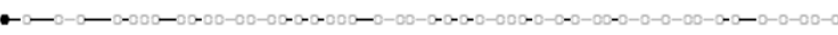

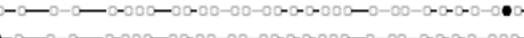

- - - -

Normal

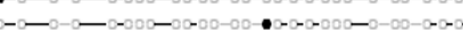

brain

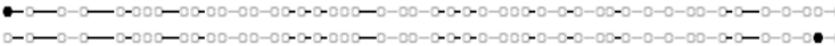

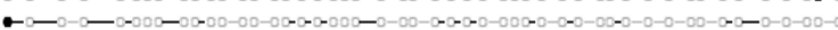

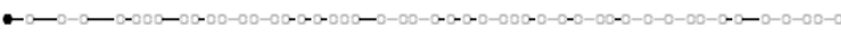

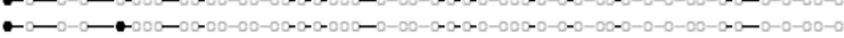

\section{Region 2}

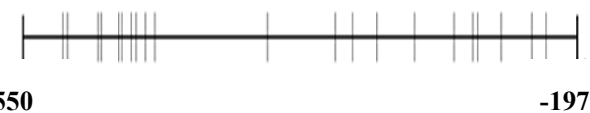

$-197$
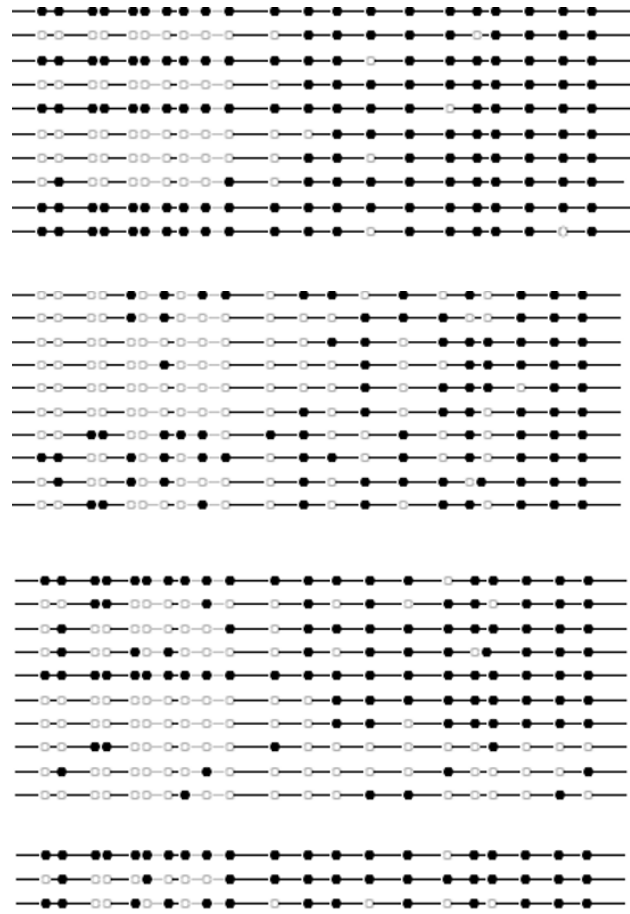

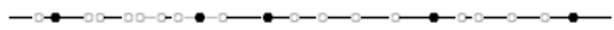

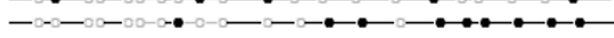

-

-

$\longrightarrow \longrightarrow 0-000-0$

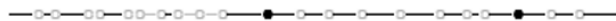

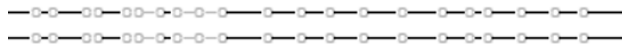

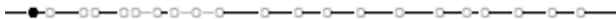

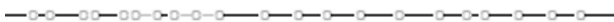

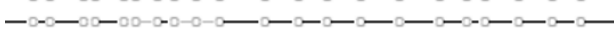

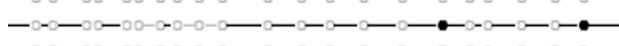

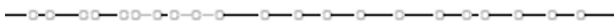

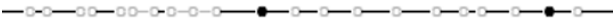

-

Figure 5

Bisulfite sequence analysis of LRRC4 promoter. Methylation status of CpG sites around the LRRC4 promoter region were analyzed in glioma cell lines (SF767 and SFI26), primary glioma (T5 and TI0) and normal brain tissue. Region I spans -934 to -529 relative to the $L R R C 4$ translation start site, including 48 CpG sites. Region 2 spans -550 to -197 relative to the $L R R C 4$ translation start site, including 2 I CPG sites. The arrow represents the region amplified by methylation-specific PCR. Each row represents an individual subclone. Open circles represent unmethylated CpGs. Filled circles represent methylated $C_{p} G s$.

To confirm that reactivation of LRRC4 mRNA expression in glioma cell lines was caused by demethylation of the LRRC4 promoter, methylation-specific PCR was used to detect methylation status changes in the LRRC4 promoter in the SF767 and SF126 cell lines after 5-Aza-dC treatment. Figure 6B shows that $5-A z a-d C$ results in the partial demethylation of LRRC4.

\section{Discussion}

Inactivation of tumor-suppressor genes plays an important role in malignant brain tumor formation and progression. Genetic mechanisms such as mutation, deletion, and structural chromosome rearrangement are known to inactivate tumor-suppressor genes. Our previous studies demonstrated that the expression of the LRRC4 gene was 


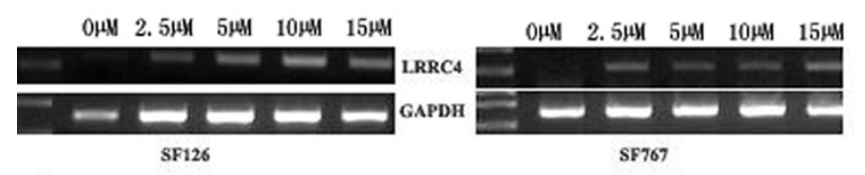

A

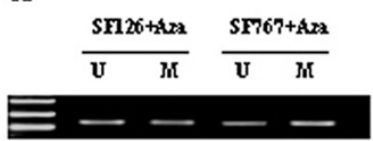

B

\section{Figure 6}

5-Aza-dC induced the expression of LRRC4 in glioma cell lines. A. Glioma cell lines SF767 and SFI 26 were treated with five different doses of 5-Aza-Dc $(0,2.5,5,10$ and I5 $\mu \mathrm{M})$, as indicated above each lane, for four days. Total RNA was isolated from control, and treated cells were then analyzed by RT-PCR using the LRRC4 primer set (top panel) and GAPDH primer set (bottom panel). B. Glioma cell lines SF767 and SFI 26 were treated with $5 \mu$ M 5-Aza-dC for four days. DNA was isolated and bisulfite-modified, and then analyzed for methylation of the LRRC4 promoter by methylationspecific PCR. The presence of a PCR product in lane $U$ indicates unmethylated $L R R C 4$. The presence of a PCR product in lane $M$ indicates the methylated LRRC4.

not only highly specific in brain tissue [26], but also a candidate tumor-suppressor gene that may be involved in the pathogenesis of malignant gliomas. However, no genetic alterations of the LRRC4 coding region were found in glioma. Based on these data, we speculated that the absence or down-regulation of LRRC4 expression in glioma may be caused by the 5' upstream regulatory sequence.

In the present study, we cloned and characterized $2375 \mathrm{bp}$ ( -2475 to -101$)$ of the 5 ' genomic region of the LRRC4 gene, which shows baseline promoter activity. Furthermore, transfection experiments using a series of 5'-deleted constructs demonstrated that the region -835 to -293 is sufficient to mediate maximal promoter activity. This region can also drive eGFP expression in the eGFPreporter plasmid (data not shown). These results suggest that we have found a function of the LRRC4 promoter.

The functional LRRC4 promoter region is a TATA- and CATT- less, high GC content region and has characteristics of a CpG island. This is consistent with brain-specific gene LRRC4 since many tissue-specific genes possess CpG islands in regulatory regions [27].

It is well known that aberrant methylation of CpG islands is one of the major modes of inactivation of tumor suppressor genes in cancer, and a growing list of genes are being identified as abnormal methylation of promoters having CpG islands [28]. Here, the LRRC4 promoter was found to be methylated in two glioma cell lines (SF126 and SF767) and all 30 primary gliomas that we have collected, but not in the normal brain tissue samples, suggesting that LRRC4 methylation is a tumor-specific event. Furthermore, there was no correlation between clinical stage, sex or age and LRRC4 methylation. Methylation of $L R R C 4$ was detected in both the early and late stages of glioma, indicating that the inactivation of the LRRC4 gene might be essential in the early development of glioma and persist through the course of development.

Treatment of SF126 and SF767 cells with 5-Aza-dC restored LRRC4 expression, which suggests that aberrant hypermethylation of the promoter is directly responsible for transcription inactivation of its expression in glioma cell lines. The molecular mechanism through which DNA methylation silences gene expression is not fully understood. DNA methylation may directly interfere with the binding of transcription factors, resulting in the transcriptional repression of the associated gene [29,30]. In addition, methyl-binding domains containing proteins (MBDs) may bind to areas of dense DNA methylation and recruit histone deacetylases and transcriptional repressor complexes, which is refractory to transcription [31]. Bisulfite sequence analysis of the $\mathrm{CpG}$ island around the LRRC4 promoter reveals dense methylation of CpG sites in glioma cell lines and tissues compared with non-tumor brain specimens (Figure 5). Taken together, these findings suggest that promoter methylation is an important mechanism in the inactivation of LRRC4 in glioma.

The present study implies that methylation-mediated inactivation of LRRC4 is involved in the initiation and development of glioma. Since LRRC4 promoter methylation is found in glioma but not in the normal brain, it may distinguish tumors from normal tissue and serve as a promising biomarker for diagnosis. Our previous study indicated that LRRC4 may be an adhesive protein or/and receptor, and it inhibited glioma invasion and metastasis [32]. Since the ability of invasion and metastasis is closely related to prognosis in glioma, LRRC4 methylation may also be a biomarker for prognosis. In addition, our findings demonstrate that methylase inhibitor can reverse LRRC4 expression in glioma, and it is possible to restore its function as tumor suppressor gene at some degree. This shows that LRRC4 may be a potential target for therapy. Of course, all of these hypotheses must be further studied using a large sample analysis.

\section{Conclusion}

In summary, methylation-mediated inactivation of LRRC4 is a frequent and glioma-specific event that may be a potential biomarker for diagnosis and prognosis, or a useful target for therapy. 


\section{Competing interests}

The authors declare that they have no competing interests.

\section{Authors' contributions}

ZPZ participated in the study design and coordination, data collection, drafting of the manuscript and DNA methylation analysis. DL contributed to patient recruitment, obtaining consent, surgical sample collection and handling, gDNA extraction and DNA methylation analysis. MHW participated in experimental design, helped to draft the manuscript and carried out data interpretation. BX and LW performed the computer analysis of the promoter region and prepared some of the constructs used throughout this work. MZ and PC were involved in cell culture, transfection experiments and analysis and carried out RT-PCR. XLL and SRS participated in drafting and revising the manuscript. GYL carried out the experiment design, manuscript drafting and revision. All authors have read and approved the final version of the manuscript.

\section{Acknowledgements}

The work was supported by National Key Project of scientific Research Program of China Grants 2006CB9I0502 and 2006CB910504, the III project of China Grant I I I-2-12, National Natural Science Foundation of China Grants 30770825 and 3060024, and Hunan Province Natural Sciences Foundations of China grant 06JJ20080.

\section{References}

I. Ohgaki $\mathrm{H}$, Kleihues P: Epidemiology and etiology of gliomas. Acta Neuropathol 2005, 109:93-108

2. Maher EA, Furnari FB, Bachoo RM, Rowitch DH, Louis DN, Cavenee WK, DePinho RA: Malignant glioma: genetics and biology of a grave matter. Genes Dev 2001, I 5:1311-1333.

3. Furnari FB, Fenton T, Bachoo RM, Mukasa A, Stommel JM, Stegh A, Hahn WC, Ligon KL, Louis DN, Brennan C, Chin L, DePinho RA Cavenee WK: Malignant astrocytic glioma: genetics, biology, and paths to treatment. Genes Dev 2007, 21:2683-2710.

4. Gömöri E, Pál J, Mészáros I, Dóczi T, Matolcsy A: Epigenetic inactivation of the hMLH gene in progression of gliomas. Diagn Mol Pathol 2007, 16:104-107.

5. Wiencke JK, Zheng S, Jelluma N, Tihan T, Vandenberg S, Tamgüney T, Baumber R, Parsons R, Lamborn KR, Berger MS, Wrensch MR, Haas-Kogan DA, Stokoe D: Methylation of the PTEN promoter defines low-grade gliomas and secondary glioblastoma. Neuro Oncol 2007, 9(3):27I-279.

6. Blanc JL, Wager M, Guilhot J, Kusy S, Bataille B, Chantereau T, Lapierre F, Larsen C], Karayan-Tapon L: Correlation of clinical features and methylation status of MGMT gene promoter in glioblastomas. J Neurooncol 2004, 68:275-283.

7. Baylin SB, Herman JG: DNA hypermethylation in tumorigenesis: epigenetics joins genetics. Trends Genet 2000, I 6: I68-174.

8. Jones PA, Baylin SB: The fundamental role of epigenetic events in cancer. Nat Rev Genet 2002, 3:415-428.

9. Costello JF, Plass C: Methylation matters. J Med Genet 200I, 38:285-303.

10. Bird AP, Wolffe AP: Methylation-induced repression - belts, braces, and chromatin. Cell 1999, 99:45I-454.

II. Costello JF: DNA methylation in brain development and gliomagenesis. Front Biosci 2003, 8: I 75-I84.

12. Alaminos M, Dávalos V, Ropero S, Setién F, Paz MF, Herranz M, Fraga MF, Mora J, Cheung NK, Gerald WL, Esteller M: EMP3, a myelinrelated gene located in the critical $19 \mathrm{q} / 3.3$ region, is epigenetically silenced and exhibits features of a candidate tumor suppressor in glioma and neuroblastoma. Cancer Res 2005 65:2565-257I.

13. Jiang Z, Li XG, Hu J, Lu DR, Zhou W, Jiang YQ, Li CY: The methylation and mRNA expression of SLC5A8 and TMSI/ASC genes in human glioma. Zhonghua Yi Xue Za Zhi 2007, 87:292-297.

14. Kim TY, Zhong S, Fields CR, Kim JH, Robertson KD: Epigenomic profiling reveals novel and frequent targets of aberrant DNA methylation-mediated silencing in malignant glioma. Cancer Res 2006, 66:7490-7450I.

15. Foltz G, Ryu GY, Yoon JG, Nelson T, Fahey J, Frakes A, Lee H, Field L, Zander K, Sibenaller Z, Ryken TC, Vibhakar R, Hood L, Madan A: Genome-wide analysis of epigenetic silencing identifies BEXI and BEX2 as candidate tumor suppressor genes in malignant glioma. Cancer Res 2006, 66:6665-6674.

16. Wang JR, Qian J, Dong L, Li XL, Tan C, Li J, Zhang BC, Zhou J, Li GY: Identification of LRRC4, a novel member of Leucine-rich Repeat (LRR) superfamily, and its expression analysis in brain tumor. Prog Biochem Biophys 2002, 29:233-239.

17. Wu MH, Huang H, Chen Q, Li D, Zheng ZY, Xiong W, Zhou YH, Li XL, Zhou M, Lu JH, Shen SR, Li GY: Leucine-rich Repeat C4 protein is Involved in Nervous Tissue Development and Neurite Outgrowth, and Induction of Glioma Cell Differentiation. Acta Biochim Biophys Sin 2007, 39:731-738.

18. Wu MH, Huang C, Gan K, Huang H, Chen Q, Ouyang J, Tang YL, Li XL, Yang YX, Zhou M, Zhou YH, Zeng ZY, Li XL, Li D, Tang K, Shen SR, Li GY: LRRC4, a Putative Tumor Suppressor Gene, Requires a Functional Leucine-rich Repeat Cassette Domain to Inhibit Proliferation of Glioma Cells In Vitro by Modulating the Extracellular Signal-regulated Kinase/Protein Kinase B/Nuclear Factor-\{kappa\}B Pathway. Mol Biol Cell 2006, I 7:3534-3542.

19. Zhang QH, Wang LL, Cao L, Peng C, Li XL, Tang K, Li W, Liao P, Wang JR, Li GY: Study of a novel brain relatively specific gene LRRC4 involved in glioma tumorigenesis suppression using the Tet-on system. Acta Biochim Biophys Sin 2005, 37:532-540.

20. Genomatix GEMSlauncher: Promoterlnspector. Search for mammalian promoters [http://www.genomatix.de/online help/help.gem/ Promoterlnspector help.html].

21. PromoterScan: Promoter region prediction. NIH Biolnformatics \& Molecular Analysis Section [http://www-bimas.cit.nih.gov/molbio/ proscan/].

22. European Bioinformatic Institute: CpGPlot program. [http:// www.ebi.ac.uk/emboss/cpgplot/].

23. University of California: MethPrimer - Design Primers for Methylation PCRs. [http://www.urogene.org/methprimer/ index.html].

24. Uhl J, Klan N, Rose M, Entian KD, Werz O, Steinhilber D: The 5lipoxygenase promoter is regulated by DNA methylation. J Biol Chem 2002, 277:4374-4379.

25. Murumägi $A$, Vähämurto $P$, Peterson $P$ : Characterization of regulatory elements and methylation pattern of the autoimmune regulator (AIRE) promoter. J Biol Chem 2003, 278: I 9784-I 9790.

26. Zhang QH, Wang JR, Fan SQ, Wang LL, Cao L, Tang K, Peng C, Li Z, Li W, Gan K, Liu Z, Li XL, Shen SR, Li GY: Expression and functional characterization of LRRC4, a novel brain-specific member of the LRR superfamily. FEBS Lett 2005, 579:3674-3682

27. Kundu TK, Rao MR: CpG islands in chromatin organization and gene expression. J Biochem 1999, I 25:217-222.

28. Herman JG, Baylin SB: Gene silencing in cancer in association with promoter hypermethylation. N Engl J Med 2003, 349:2042-2054

29. Issa JP: Epigenetic variation and human disease. J Nutr 2002, I 32:2388S-2392S.

30. Jones PA, Baylin SB: The fundamental role of epigenetic events in cancer. Nat Rev Genet 2002, 3:4I5-428.

31. Laird PW: Cancer epigenetics. Hum Mol Genet 2005, I4:R65-R76.

32. Wu MH, Gan K, Huang C, Tang YX, Chen Q, Tang K, Li XL, Shen SR, Li GY: LRRC4 controls in vitro invasion of glioblastoma cells through inhibiting RPTP-zeta expression. I Neurooncol 2006, 80: $133-142$

33. Louis DN, Ohgaki H, Wiestler OD, Cavenee WK, Burger PC, Jouvet A, Scheithauer BW, Kleihues P: The 2007 WHO classification of tumours of the central nervous system. Acta Neuropathol 2007, I | 4:97-109. 